\title{
Comparison on the Pharmacokinetics and Weight Reduction of Clobenzorex Slow Release and Immediate Release Formulations in Obese Patients
}

\author{
Federico Argüelles-Tello ${ }^{1}$, Miriam del C. Carrasco-Portugal ${ }^{2}$, Norma A. Carrasco-Portugal ${ }^{3}$, \\ José Carlos Aguilar-Carrasco ${ }^{1,4}$, Selene I. Patiño-Camacho ${ }^{1}$, Cecilia Fernández del Valle ${ }^{1}$, \\ Gerardo Reyes-Garcia $^{1}$, Francisco J. Flores-Murrieta ${ }^{1,2}$
}

\begin{abstract}
${ }^{1}$ Sección de Estudios de Posgrado e Investigación, Escuela Superior de Medicina del Instituto Politécnico Nacional, México City, México; ${ }^{2}$ Unidad de Investigación en Farmacología, Instituto Nacional de Enfermedades Respiratorias Ismael Cosío Villegas, México City, México; ${ }^{3}$ Centro de Estudios Biofarmacéuticos, México City, México; ${ }^{4}$ Instituto Nacional de Perinatología, México City, México.

Email: fjfloresmurrieta@yahoo.com.mx, francisco.floresmurrieta@gmail.com
\end{abstract}

Received January $18^{\text {th }}, 2013$; revised February $23^{\text {rd }}, 2013$; accepted April $4^{\text {th }}, 2013$

Copyright (C) 2013 Federico Argüelles-Tello et al. This is an open access article distributed under the Creative Commons Attribution License, which permits unrestricted use, distribution, and reproduction in any medium, provided the original work is properly cited.

\begin{abstract}
Clobenzorex is an anorexigenic drug that is widely used in Mexico for the treatment of obesity, since it helps to reduce body weight. This drug is available as immediate release capsules. To improve compliance to treatment, it was developed a new slow release formulation. In order to establish its usefulness, oral pharmacokinetics and weight reduction of slow release and immediate release formulations of clobenzorex in obese patients were compared. Sixty patients with a BMI higher than $27 \mathrm{~kg} / \mathrm{m}^{2}$ were included in the study. Two groups of 30 patients were formed, one of them received 30 $\mathrm{mg}$ immediate release formulation b.i.d. and the other group received one $60 \mathrm{mg}$ slow release formulation once a day, since under this scheme these formulations are prescribed. Blood samples were obtained at selected times during the first day and once weekly during 4 weeks. After the last dose, samples were obtained at selected times during $48 \mathrm{~h}$. Plasma levels were determined by HPLC-MS/MS and pharmacokinetic parameters were obtained. Reduction in $\mathrm{C}_{\max }$ due to increased $t_{\max }$, as well as, increased half-life were observed with the slow release formulation in comparison with immediate release formulation. Although lower plasma levels of clobenzorex were reached with the slow release formulation, reduction of body weight was similar with both products. Based on the results, it was concluded that slow release formulation of clobenzorex is an adequate formulation of clobenzorex, since pharmacokinetics and effects observed are compatible with a once a day administration.
\end{abstract}

Keywords: Clobenzorex; Pharmacokinetics; Slow Release Formulation

\section{Introduction}

Clobenzorex is a sympathomymethic amine with low addictive activity and with anorexygenic activity that is widely used in Mexico for the treatment of obesity. It is recommended for patients with BMI higher than 30 $\mathrm{kg} / \mathrm{m}^{2}$ who did not respond to diet and exercise. Clobenzorex produces its anorexygenic activity by increasing noradrenaline levels; this noradrenaline reduces appetite by activating alpha- 4 and beta- 1 adrenergic receptors in hypothalamus $[1,2]$.

Clobenzorex has a pKa of 8.8 to 10.4 and after oral administration, clobenzorex is well absorbed in gastrointestinal tract, reaching the maximal concentration in about 1 to 1.5 hours and plasma levels varies between 8 to $47 \mathrm{ng} / \mathrm{ml}[3,4]$. Clobenzorex is widely distributed in tissues, in fact, levels reached in spinal cord fluid are similar to those reached in plasma. It is bound to plasma proteins in about $15 \%-30 \%$ and is metabolized mainly to 4-hydroxyclobenzorex. Half-life of clobenzorex is widely variable, since values observed are from 1 to 17 hours.

Clobenzorex is eliminar palabra used as an immediate release formulation at dosage regimes of $30 \mathrm{mg}$ b.i.d. [5], however, peak concentration reached may produce a high incidence of side effects and may increase the probability of drug abuse. In order to make the treatment more suitable and reducing both, side effects and probability of 
drug abuse, a slow release formulation was developed. The purpose of this study was to compare the pharmacokinetics and the reduction of body weight of these formulations in overweighted and obese patients under treatment for one month.

\section{Subjects, Material and Methods}

Sixty patients (45 women, 15 men) with body mass index higher than $27 \mathrm{~kg} / \mathrm{m}^{2}$ were included in the study. All subjects were evaluated by clinical history, medical examination and suitable laboratory tests, in order to discard any comorbility. All patients that met the inclusion criteria were randomly assigned to one of two groups of thirty subjects. Comparison of demographic data of treatment groups are given in Table 1. Group one received treatment with immediate release capsules of 30 $\mathrm{mg}$ b.i.d. for four weeks, whereas, group two received slow release tablet of $60 \mathrm{mg}$ once a day for the same period. These treatment schemes were employed, since immediate release formulation was prescribe under these conditions and slow release formulation was intend to be used once a day. Once the subjects were assigned to the treatment group they were hospitalized to start the treatment with clobenzorex and after the first dose, blood samples were collected at $0,0.25,0.5,0.75,1,1.25,1.5$, $2,3,4,5,6,8,10,12$ and $24 \mathrm{~h}$. Plasma was obtained by centrifugation and stored frozen at $-80^{\circ} \mathrm{C}$ until analyzed. After obtaining the last blood sample, patients were provided of medication enough for a week and allowed to continue their normal activities. They were instructed to come back to the clinical facilities after a week for evaluation of anthropometric measurements and obtaining through plasma sample. This procedure was repeated on week 2, 3 and 4. After the last dose administration, subjects were maintained in the clinical facilities and blood samples were obtained at $0,0.25,0.5,0.75,1,1.25$, $1.5,2,3,4,5,6,8,10,12,24$ and $48 \mathrm{~h}$. Plasma was obtained and stored as described above. A final evaluation of anthropometric parameters was carried out.

\subsection{Determination of Clobenzorex in Plasma}

Plasma levels of clobenzorex were determined by a highperformance liquid chromatographic method coupled to MS/MS detection. Briefly, $0.5 \mathrm{ml}$ were placed in $15 \mathrm{ml}$ conical tubes and added with $12.5 \mathrm{ng}$ of paroxetine as internal standard and $4 \mathrm{ml}$ of methyl-tert-butyl ether. This mixture was agitated in a vortex mixer at maximal speed for 1 minute and centrifuged at $3500 \mathrm{rpm}$ for 10 minutes. Upper layer was transferred to another tube and evaporated to dryness at $35^{\circ} \mathrm{C}$ under nitrogen stream. Dry residue was redissolved in $200 \mu \mathrm{l}$ of mobile phase (see under) and $10 \mu \mathrm{l}$ aliquots were injected into the chromatographic system.

The chromatographic system was formed by an acquity ultra performance liquid chromatograph (Waters Assoc, Milford, MA, USA) coupled to a API-3200 mass/ mass spectrometer (Applied Biosystems, Inc.). Separation of compounds was carried out using an acquity UPLC BEH Phenyl $100 \mathrm{~mm}$ length $\times 2.1 \mathrm{~mm}$ i.d. of 1.7 $\mu \mathrm{m}$ particle size column eluted with a mixture of methanol with $0.3 \%$ formic acid solution $(75: 25, \mathrm{v} / \mathrm{v})$ at a flow rate of $0.2 \mathrm{ml} / \mathrm{min}$. The sample were subsequently analyzed by mass spectrometry with an ESI source and then quantified by multiple reaction monitoring (MRM). The precursor-product ion combinations of $\mathrm{m} / \mathrm{z} 260.1 \rightarrow 91.1$ and $\mathrm{m} / \mathrm{z} 330.1 \rightarrow 70.0$ was used to quantify clobenzorex and internal standard, respectively. Under these conditions, clobenzorex retention time is $1.22 \mathrm{~min}$ and paroxetine is $1.29 \mathrm{~min}$ and no interferences occurs at this time. Typical chromatograms obtained with the employed method are shown in Figure 1.

Under these conditions, the method was linear in the range of 0.05 to $100 \mathrm{ng} / \mathrm{ml}$, with an intra- and inter-day accuracy of $95.33 \%$ and the coefficient of variation was lower than $13 \%$.

\subsection{Pharmacokinetic and Statistical Analysis}

Individual plasma level-time curves were constructed and maximal concentration $(\mathrm{Cmax})$ and time to reach this

Table 1. Demographic data of subjects enrolled in the study of comparison of pharmacokinetics and body weight after administration of immediate release and slow release formulations of clobenzorex. Data are expressed as mean \pm SD. No statistically significant difference in any parameter was obtained.

\begin{tabular}{ccc}
\hline & Clobenzorex slow release formulation $(60 \mathrm{mg}) \mathrm{n}=30$ & Clobenzorex immediate release formulation $(30 \mathrm{mg}) \mathrm{n}=30$ \\
\hline Age (years) & $35.03 \pm 8.97$ & $33.97 \pm 11.43$ \\
Sex $(\mathrm{F} / \mathrm{M})$ & $23 / 7$ & $22 / 8$ \\
Weight $(\mathrm{kg})$ & $90.51 \pm 15.88$ & $84.07 \pm 15.51$ \\
Waist $(\mathrm{cm})$ & $105.9 \pm 13.24$ & $100.76 \pm 12.18$ \\
Height $(\mathrm{m})$ & $1.642 \pm 0.078$ & $1.607 \pm 0.090$ \\
BMI $\left(\mathrm{kg} / \mathrm{m}^{2}\right)$ & $33.47 \pm 4.77$ & $32.54 \pm 4.08$ \\
\hline
\end{tabular}



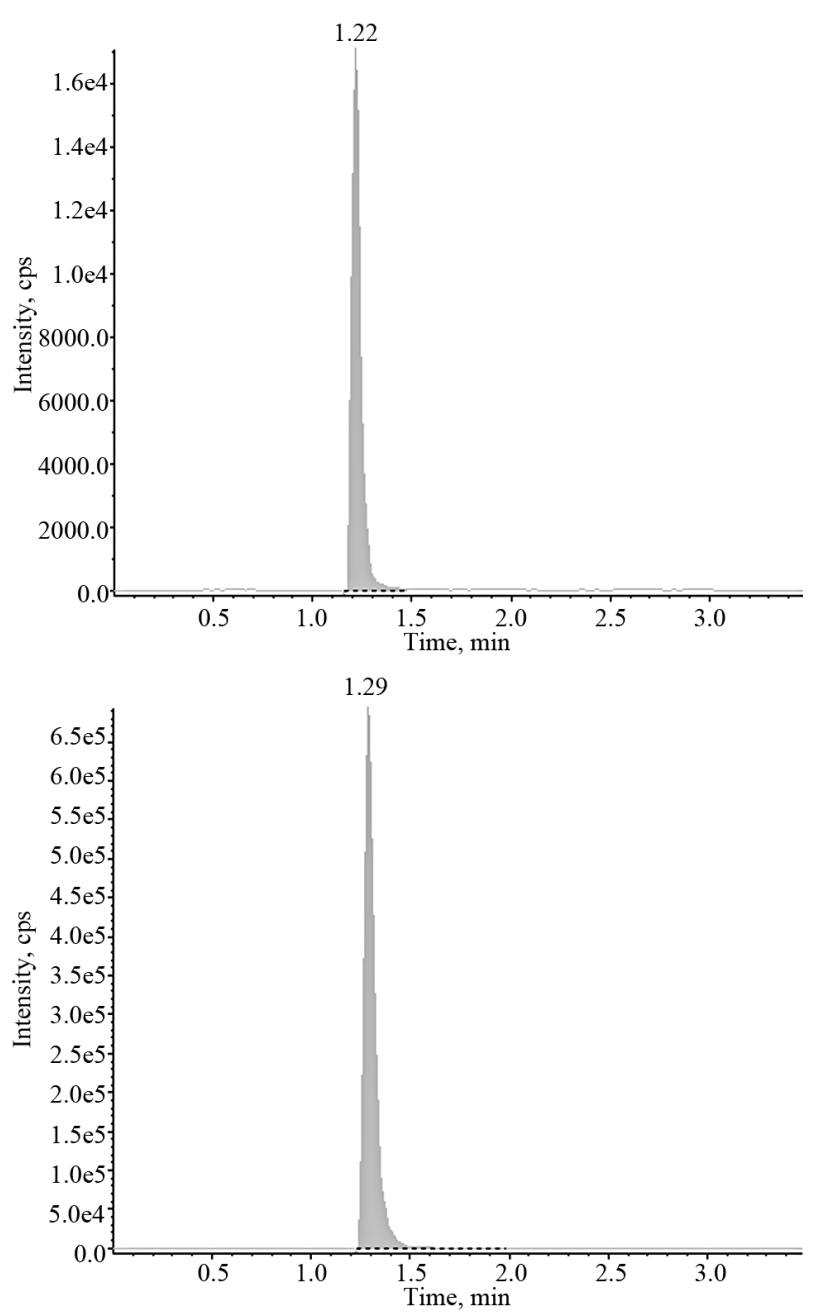

Figure 1. Typical chromatograms obtained after injection of the extract of a blank plasma spiked with both, $25 \mathrm{ng} / \mathrm{ml}$ clobenzorex (upper part) and $25 \mathrm{ng} / \mathrm{ml}$ of the internal standard (lower part).

maximum (Tmax) were directly obtained of such curves. Area under the plasma concentration against time curve until the last sampling time (AUClast) was obtained by the trapezoidal rule, extrapolation to infinity was carried out by dividing the last concentration observed by the elimination constant rate value [6]. Comparison of pharmacokinetic parameters was carried out by student-t-test. On the other hand, weight and body mass index reduction with the formulations tested were compared by analysis of variance followed by Tukey test.

\section{Results}

Figure 2 shows plasma level-time curves after the first administration of clobenzorex as a $30 \mathrm{mg}$ immediate release capsule and a $60 \mathrm{mg}$ slow release tablet. It can be seen that lower concentrations with the slow release formulation are reached, however, long lasting concen-

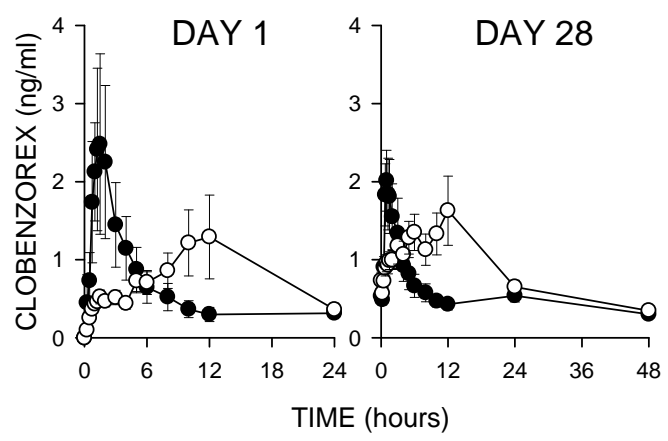

Figure 2. Plasma concentration against time curves after administration of clobenzorex $30 \mathrm{mg}$ immediate release capsule b.i.d (dark circles) and $60 \mathrm{mg}$ slow release tablet (open circles) after the first dose (left side) and after the day 28 (right side). Data are expressed as mean \pm s.e.m. of 30 subjects per group.

trations were observed with such formulation. Pharmacokinetic parameters obtained are shown in Table 2 . It is important to note that although frequency of administration was different with the formulations tested, through levels are similar, as shown in Table 3.

Table 4 shows the comparison of the reduction of body weight and body mass index in the subjects submitted to both treatments. It is clearly shown that no statistically significant difference was observed in the rate of loss of weight during the treatment with both formulations. It is important to note that both treatments were well tolerated. Only 7 mild events were observed with immediate release formulation and 10 mild events with the slow release formulation, being the most frequent adverse events headache, dizziness and nausea.

\section{Discussion}

Pharmacokinetics and weight reduction of clobenzorex administered as $30 \mathrm{mg}$ immediate release capsule b.i.d. and $60 \mathrm{mg}$ slow release tablet once a day were compared. Although clobenzorex is one of the best seller drugs in Mexico, so far, no information about its pharmacokinetics in Mexicans is available.

When pharmacokinetics of the two formulations was compared, it was observed that through levels reached with both schemes are similar, but maximal concentration was reduced. This last was expected for a slow release formulation. An important aspect is that drug abuse potential, as well as, side effects are more dependent than time of exposition to the drug and the profile observed with the slow release formulation are compatible with this fact. On the other hand, reduction of weight due to loss of appetite seems not to depend on the $\mathrm{C}_{\max }$ reached, but the time of exposure to the drug. Since both formulations reached similar through levels, it may be possible that reduction of body weight and therefore, body mass 
Table 2. Comparison of the pharmacokinetic parameters obtained after administration of clobenzorex immediate release and slow release formulations. Data are expressed as mean \pm s.e.m. of thirty subjects per group.

\begin{tabular}{cccc}
\hline Parameter & Slow release formulation $(60 \mathrm{mg})$ & Immediate release formulation $(30 \mathrm{mg})$ & $\mathrm{p}$-value \\
\hline $\mathrm{C}_{\max }(\mathrm{ng} / \mathrm{ml})$ & $1.777 \pm 0.514$ & $3.429 \pm 1.187$ & $<0.01$ \\
$\mathrm{~T}_{\max }(\mathrm{h})$ & $6.183 \pm 0.764$ & $1.458 \pm 0.187$ & $<0.001$ \\
$\mathrm{AUC}(\mathrm{ng} \cdot \mathrm{h} / \mathrm{ml})$ & $17.770 \pm 4.529$ & $11.076 \pm 3.917$ & $<0.05$ \\
$\mathrm{~T}_{1 / 2}(\mathrm{~h})$ & $15.002 \pm 4.946$ & $3.118 \pm 0.271$ & $<0.001$ \\
\hline
\end{tabular}

Table 3. Through plasma levels reached after administration of $30 \mathrm{mg}$ immediate release capsule of clobenzorex b.i.d. and 60 mg slow release tablet once a day. Data are expressed as mean \pm s.e.m. of 30 subjects per group. No statistically significant difference between treatments was observed.

\begin{tabular}{ccc}
\hline $\begin{array}{c}\text { Day of } \\
\text { treatment }\end{array}$ & $\begin{array}{c}\text { Concentration observed }(\mathrm{ng} / \mathrm{ml}) \text { after slow release formulation } \\
(60 \mathrm{mg})\end{array}$ & $\begin{array}{c}\text { Concentration observed }(\mathrm{ng} / \mathrm{ml}) \text { after immediate release formulation } \\
(30 \mathrm{mg})\end{array}$ \\
\hline 7 & $1.0714 \pm 0.1962$ & $1.0897 \pm 0.1799$ \\
14 & $0.9282 \pm 0.1361$ & $1.2280 \pm 0.2494$ \\
21 & $0.9363 \pm 0.1236$ & $1.3314 \pm 0.2357$ \\
28 & $1.3075 \pm 0.3283$ & $1.4925 \pm 0.4671$ \\
\hline
\end{tabular}

Table 4. Cummulative body mass index and weight reduction during the treatment with clobenzorex $60 \mathrm{mg}$ slow release tablet administered once a day and $30 \mathrm{mg}$ immediate release capsule administered b.i.d. Data are expressed as mean \pm s.e.m. No statistically significant difference between treatments was observed.

\begin{tabular}{ccccc}
\hline \multirow{2}{*}{ Day of treatment } & \multicolumn{2}{c}{ Body weight reduction $(\mathrm{kg})$} & \multicolumn{2}{c}{ BMI reduction $\left(\mathrm{kg} / \mathrm{m}^{2}\right)$} \\
\cline { 2 - 5 } & Immediate release capsule & Slow release tablet & Immediate release capsule & Slow release tablet \\
\hline 7 & $1.75 \pm 0.28$ & $1.44 \pm 0.21$ & $0.67 \pm 0.11$ & $0.53 \pm 0.08$ \\
14 & $1.96 \pm 0.29$ & $1.90 \pm 0.26$ & $0.74 \pm 0.11$ & $0.70 \pm 0.10$ \\
21 & $2.33 \pm 0.38$ & $2.44 \pm 0.33$ & $0.90 \pm 0.14$ & $0.91 \pm 0.13$ \\
28 & $2.65 \pm 0.38$ & $2.57 \pm 0.36$ & $1.03 \pm 0.14$ & $0.95 \pm 0.14$ \\
\hline
\end{tabular}

index will be similar. This was the case, since rate of reduction of body weight and body mass index was similar with the evaluated formulations.

In conclusion, clobenzorex slow release tablet shows an adequate pharmacokinetic profile and therefore, it is a good alternative for supporting body weight reduction, additional to diet and exercise, since its anorexigenic activity with this formulation should be mantained with a once a day administration.

\section{Acknowledgments}

Authors kindly acknowlegde Productos Medix, S. A. de C. V., Mexico City, Mexico for the formulations gifted in this study.

\section{REFERENCES}

[1] B. Glasson, A. Benakis and M. Thomasset, "Localisation, Distribution, Excretion et Metabolisme d'eun Nouveau Medicament Anorexigene Marqué au C14: Le Cholhydrate de Clobenzorex," Arzneim-Forsch, Vol. 21, 1971, pp. 1985-1992.

[2] S. Valtier and J. T. Cody, "Differentiation of Clobenzorex Use from Amphetamine Abuse Using the Metabolite 4-Hydroxyclobenzorex," Journal of Analytical Toxicology, Vol. 24, No. 7, 2000, pp. 606-613. doi:10.1093/jat/24.7.606

[3] K. L. Baden, S. Valtier and J. T. Cody, "Metabolic Production of Amphetamine Following Multidose Administration of Clobenzorex," Journal of Analytical Toxicology, Vol. 23, No. 6, 1999, pp. 511-517. doi:10.1093/jat/23.6.511

[4] J. T. Cody and S. Valtier, "A Gas Chromatography-Mass Spectrometry Method for the Quantitation of Clobenzorex," Journal of Analytical Toxicology, Vol. 23, 1999 pp. 603-608.

[5] Goodman and Gilman, "Las Bases Farmacológicas de la Terapéutica,” Décima Edición, McGraw Hill, New York, 2006, pp. 223-275.

[6] M. Rowland and T. N. Tozer, "Clinical Pharmacokinetics. Concepts and Applications," 3rd Edition, Lea \& Febiger, Philadelphia, London, 1995. 